Article

\title{
Geometrical Phase Optical Components: Measuring Geometric Phase without Interferometry
}

\author{
Oriol Arteaga ${ }^{1,2, *(1)}$ and Hana Bendada ${ }^{3}$ \\ 1 Department Física Aplicada, Universitat de Barcelona, 08028 Barcelona, Spain \\ 2 Institute of Nanoscience and Nanotechnology (IN2UB), Universitat de Barcelona, 08028 Barcelona, Spain \\ 3 Institute of Optics and Precision Mechanics, Ferhat Abbas University of Setif, Setif 19000, Algeria; \\ bendadahana@univ-setif.dz \\ * Correspondence: oarteaga@ub.edu
}

Received: 22 August 2020; Accepted: 26 September 2020; Published: 29 September 2020

\begin{abstract}
Optical components that are based on Pancharatnam-Berry phase feature a polarization-dependent diffraction that can be used to fabricate lenses and gratings with unique properties. In recent years, the great progress made in the fabrication of the metasurfaces that are required for these optical components has lowered their cost and has made them widely available. One of the often-overlooked properties of optical components based on geometrical phases (GPs) is that, contrary to dynamical phases, their phase can be measured while using a polarimetric technique without the need to resort to interferometry methods. This is possible because the Pancharatnam-Berry phase is not controlled by an optical path difference; it results from a space variant polarization manipulation. In this work, we apply Mueller matrix microscopy in order to measure the geometrical phase of GP lenses and polarization gratings. We show that a single space resolved Mueller matrix measurement with micrometric resolution is enough to obtain a full characterization phase-profile of these GP-based optical components and evaluate their performance.
\end{abstract}

Keywords: geometric phase; structured light; polarimetry

\section{Introduction}

In the last years, the interest in the concept of "structured light", which is light featuring a relatively complex spatial structure, has been continuously growing [1]. In standard optical images, the spatial structure affects only the intensity distribution of light, but a growing number of techniques and applications are based on controlling the phase and polarization distributions. The propagation behaviour of light is determined by its wavefront structure, which can be controlled by the phase distribution introduced by optical elements as it happens for example with the simple refractive optical lenses, but also in other more recently engineered optical elements, such as spatial light modulators, holograms, plasmonic or dielectric metasurfaces, etc. [2-4]. In this paper, we focus on the study of geometrical phase (GP) optical elements, in which the wavefront structure of light is controlled by phase retardation (birefringent effects), which can be made independent from the propagation length.

The first observation of a geometric phase in optics was made by S. Pancharatnam when studying the interference of polarized beams produced by crystal plates [5]. He noticed a phase shift arising when the polarization of a light was varied in a cyclic manner that corresponded to half of the solid angle subtended by the polarization cycle on the Poincaré sphere. In fact, the Poincare sphere representation of polarization states was crucial for S. Pancharatnam to recognize the geometric nature of this phase. For many years, the work of Pancharatnam went almost unnoticed and received little attention but, decades later, it gained the deserved recognition when M.V.Berry showed the equivalence 
between Pancharatnam's geometric phase in polarization optics and the broader phenomenon of the acquired phase in the adiabatic evolution of a state in quantum mechanics [6,7].

Let us start considering the Jones matrix of a half wave plate (fast axis horizontal) in order to introduce in a straightforward way the working principle of GP optical components:

$$
\mathbf{J}=e^{-\pi i / 2}\left(\begin{array}{cc}
1 & 0 \\
0 & -1
\end{array}\right)
$$

The rotated half wave plate by an angle $\phi$ is

$$
\mathbf{J}^{\prime}=\mathbf{R}(-\phi) \mathbf{J} \mathbf{R}(\phi)=e^{-\pi i / 2}\left(\begin{array}{cc}
\cos 2 \phi & \sin 2 \phi \\
\sin 2 \phi & -\cos 2 \phi
\end{array}\right)
$$

where $\mathbf{R}(\phi)$ is the usual $2 \times 2$ rotation matrix

$$
\mathbf{R}(\phi)=\left(\begin{array}{cc}
\cos \phi & \sin \phi \\
-\sin \phi & \cos \phi
\end{array}\right)
$$

For circularly polarized light with Jones vector $\mathbf{E}_{ \pm}=\left(\begin{array}{ll}1 & \pm i\end{array}\right)^{T}$, the Jones vector of the exit light can be calculated as

$$
\mathbf{J}^{\prime} \mathbf{E}_{ \pm}=e^{-\pi i / 2}\left(\begin{array}{cc}
\cos 2 \phi & \sin 2 \phi \\
\sin 2 \phi & -\cos 2 \phi
\end{array}\right)\left(\begin{array}{c}
1 \\
\pm i
\end{array}\right)=e^{-\pi i / 2} e^{ \pm 2 \phi i}\left(\begin{array}{c}
1 \\
\mp i
\end{array}\right)
$$

where the phase factor contains two parts: one that only depends on the amount of retardance and the other that is twice the orientation angle $\phi$. If we consider a situation in which the angle of rotation $\phi$ is not a constant but changes from point to point in space (i.e. $\phi(x, y)$ ), the wave will be subjected to a transversely inhomogeneous polarization transformation that will produce a waveform reshaping, depending on the polarization of light, opening the possibility for the so-called Pancharatnam-Berry phase optical elements. They were first proposed by Bharandi [8] and later by Bomzon et al. [9]. The experimental realizations did not came out until a few years later [10,11].

In this paper, we use optical polarimetry in the visible spectral range, in particular Mueller matrix spectroscopy and Mueller matrix microscopy, in order to measure the phase and evaluate the performance of commercially available GP lenses and gratings. The manuscript is organized as follows: after this introduction, Section 2 describes further theoretical details regarding GP optical components that are needed to understand our polarimetry study. Section 3 provides the experimental details of our polarimetry measurements. The main results that can be extracted from our experimental measurements are presented and discussed in Section 4 . Finally, Section 5 gives the concluding remarks.

\section{Theoretical Aspects}

\subsection{Closed Path vs. Open Path in the Poincaré Sphere}

The phase term $\pm 2 \phi i$ that appears in Equation (4) is often directly described as the Panchratnam-Berry geometrical phase, however the vector-matrix transformation given by Equation (4) is not describing any closed loop in Poincaré sphere, as originally described by Pancharatnam [5], because the initial and final polarization states are different (output states are orthogonal to input states). This transformation corresponds to a path from the north pole to the south pole (or vice-versa) of the Poincaré sphere through a geodesic arc (a meridian) with an azimuth angle $2 \phi$ with respect to a reference direction, as illustrated in Figure 1a. 
The overall path can be closed by, for example, applying twice the transformation that is given by the Jones matrix $\mathbf{J}^{\prime}$ :

$$
\mathbf{J}^{\prime} \mathbf{J}^{\prime} \mathbf{E}_{ \pm}=e^{-\pi i} \mathbf{E}_{ \pm},
$$

where now the closed loop (note that the initial and final polarization states coincide) is dividing the Poincare sphere in two hemispheres. The Pancharatnam-Berry phase is $\pi$ and the solid angle is $2 \pi$ (the solid angle of half sphere). Figure $1 \mathrm{~b}$ presents this case.
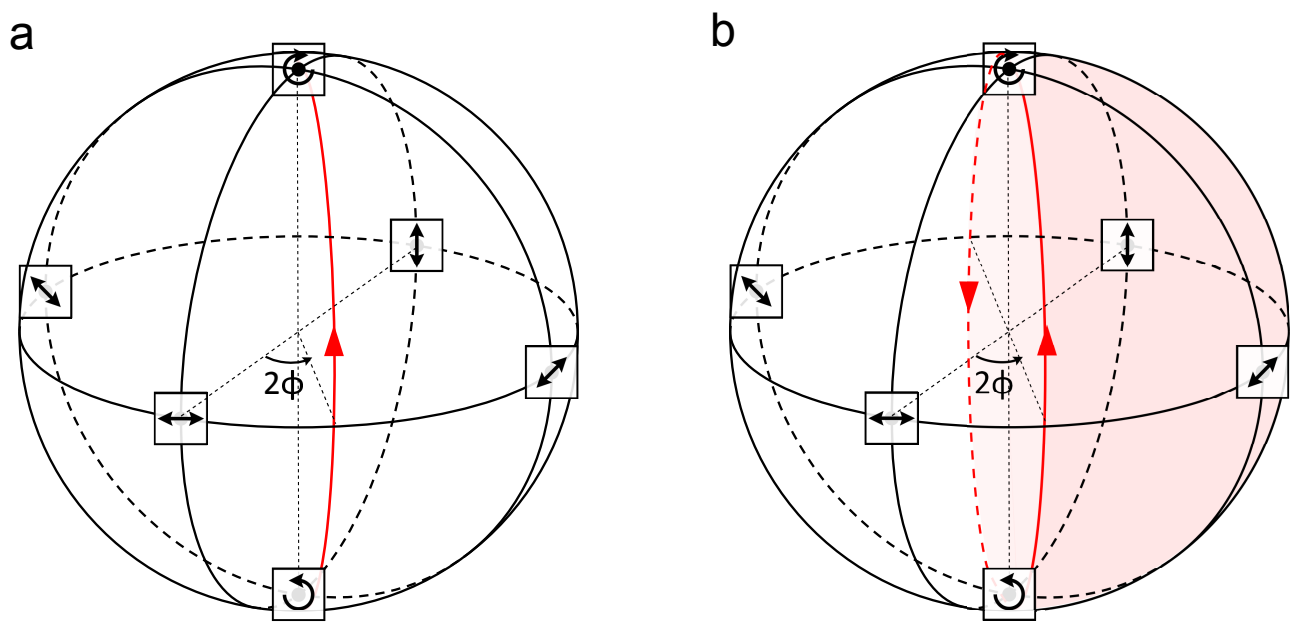

Figure 1. Poincaré sphere representation of the transformations $\mathbf{J}^{\prime} \mathbf{E}_{-}$(a) and $\mathbf{J}^{\prime} \mathbf{J}^{\prime} \mathbf{E}_{-}$(b). Note that only panel $b$ is representing a closed loop.

The pioneering works from Banhari [8] and Roux [10], describing a GP lens, assumed a particular experimental situation in order to consider a closed path in the Poincare sphere that has a dependence of $\phi$ : the incident light was linearly polarized at $45^{\circ}$, then they used a quarter-wave plate with a fixed orientation to turn the linearly polarized light into right-handed circular polarized light, which was turned into left-handed circular polarized light with a half-wave plate with varying orientation (the GP lens). Finally, the left-handed circular polarized light was turned back into the original linear polarization with another quarter-wave plate, thereby closing the path on the Poincare sphere. Thus, their description of the experiment includes not only the GP optical element, but some additional quarter wave plates. However, the effect of GP optical components becomes manifest without being restricted to a closed path like this. The simplest manifestation of geometrical phase optical components is already observed in Equation (4): two transformations that share the same initial and final states introduce optical phases of opposite sign, depending on the handedness of the incoming polarization.

\subsection{A Geometrical Phase Optical Component Based on a General Retarder}

In a real geometrical phase optical components, the optical element may not be a perfect half-wave plate retarder, as we have considered in the introduction, so Equation (1) may have to be replaced by the Jones matrix of a general retarder. Therefore, we will now study now how the effect is manifested for a general retarder with linear retardance $L$ (with fast axis horizontal) and circular retardance $C$. The Jones matrix for such optical element is $[12,13]$ :

$$
\mathbf{J}_{g}=\left(\begin{array}{cc}
\cos \frac{T}{2}-\frac{i L}{T} \sin \frac{T}{2} & \frac{C}{T} \sin \frac{T}{2} \\
-\frac{C}{T} \sin \frac{T}{2} & \cos \frac{T}{2}+\frac{i L}{T} \sin \frac{T}{2}
\end{array}\right),
$$

where $T=\sqrt{L^{2}+C^{2}}$ is representing the overall retardance. In this work, $L, C$, and $T$ are considered to be real quantities, which indicates that they include retarding properties only; in case we wanted to include some diattenuating properties-, $L, C$, and $T$ should be complex valued. 
Following the same steps as in Equation (2), we rotate the retarder:

$$
\mathbf{J}_{g}^{\prime}=\mathbf{R}(-\phi) \mathbf{J}_{g} \mathbf{R}(\phi)=\left(\begin{array}{cc}
\cos \frac{T}{2} & \frac{C}{T} \sin \frac{T}{2} \\
-\frac{C}{T} \sin \frac{T}{2} & \cos \frac{T}{2}
\end{array}\right)-i \frac{L}{T} \sin \frac{T}{2}\left(\begin{array}{cc}
\cos 2 \phi & \sin 2 \phi \\
\sin 2 \phi & -\cos 2 \phi
\end{array}\right),
$$

where the resulting rotated Jones matrix has been factorized into two terms. Note that the first term is independent of the rotation angle $\phi$ that is fully embedded in the second term. The effect of this general rotated retarder to the circularly polarized light that is transmitted can be calculated as

$$
\mathbf{J}_{g}^{\prime} \mathbf{E}_{ \pm}=\left(\begin{array}{c}
\cos \frac{T}{2} \pm i \frac{C}{T} \sin \frac{T}{2} \\
-\frac{C}{T} \sin \frac{T}{2} \pm i \cos \frac{T}{2}
\end{array}\right)-e^{ \pm 2 \phi i} \frac{L}{T} \sin \frac{T}{2}\left(\begin{array}{c}
1 \\
\mp i
\end{array}\right) .
$$

The first term is not affected by any phase term, so it will not alter the waveform. This contribution is known as the zero-order leakage wave. The second term, which has the opposite handedness than the input, is the only one that can contribute to the wavefront reshaping, since it is affected by a PB phase of $\pm 2 \phi$.

Note that, if $C=0$ and $L=\pi$, the first term in Equations (7) and (8) vanishes and we recover the "ideal" case introduced in Equation 4 that does not have any leakage wave. A different situation arises when $C=0$ and $L \neq \pi$, in this case there is still a leakage wave but it has the same polarization as the input wave, since the first matrix term in Equation (7) becomes the identity, thus indicating that there is no alteration in the polarization. This case is discussed in Ref. [14]. We may consider a third particular case, when $C \neq 0$ and $L=\pi$, which, in principle, is not substantially different from the general situation, because there is still a leakage wave and there is a change of polarization with respect to the input, but we will discuss this case in more detail in Section 4.4.

In what follows, we focus our attention in the second term of Equation (7). It is possible to rewrite it as:

$$
\left(\begin{array}{cc}
\cos 2 \phi & \sin 2 \phi \\
\sin 2 \phi & -\cos 2 \phi
\end{array}\right)=\frac{e^{-2 \phi i}}{2}\left(\begin{array}{cc}
1 & -i \\
i & 1
\end{array}\right)\left(\begin{array}{cc}
1 & 0 \\
0 & -1
\end{array}\right)+\frac{e^{2 \phi i}}{2}\left(\begin{array}{cc}
1 & i \\
-i & 1
\end{array}\right)\left(\begin{array}{cc}
1 & 0 \\
0 & -1
\end{array}\right)
$$

where we have factorized the initial matrix into two different terms, each one of them associated with a geometrical phase $\pm 2 \phi$. Interestingly, each one of terms is the product of the Jones matrix of a right circular polarizer, $\frac{1}{2}\left(\begin{array}{cc}1 & i \\ -i & 1\end{array}\right)$, or a left circular polarizer, $\frac{1}{2}\left(\begin{array}{cc}1 & -i \\ i & 1\end{array}\right)$, by the Jones matrix of a half-wave retarder [Equation (1)]. The presence of the circular polarizer Jones matrices indicates that, regardless of the incoming polarization, the waves with a GP of $\pm 2 \phi$ will always be circularly polarized, so the transformation of a general incoming wave can be summarized, as follows $[15,16]$ :

$$
\mathbf{E}_{\text {in }} \rightarrow A_{\text {leak }} \mathbf{E}_{\text {leak }}+A_{+} e^{2 \phi i} \mathbf{E}_{+}+A_{-} e^{-2 \phi i} \mathbf{E}_{-},
$$

where the amplitude $A_{i}$ terms indicate how the incoming energy is redistributed between the three waves: leakage, primary (arbitrarily associated with right circular polarization), and conjugate (arbitrarily associated with left circular polarization). An important feature of GP optical elements is that there are not higher-order parasitic waves caused by diffraction. If the GP optical element acts as a pure retarder and there are no losses the amplitudes satisfy $A_{\text {leak }}^{2}+A_{+}^{2}+A_{-}^{2}=1$. It is also important to remark that the circular polarization associated with $\mathbf{E}_{+}$and $\mathbf{E}_{-}$is kept, regardless of the input polarization and the retarding properties of the optical element, but the polarization of the leakage wave, as given by $\mathbf{E}_{\text {leak }}$, will depend on both the input polarization $\mathbf{E}_{\text {in }}$ and the values of $C$ and $L$.

Equation (10) shows that GP optical elements effectively work as circular polarization beam splitters, separating any incoming beam into two circularly polarized beams of opposite handedness, as schematically shown in Figure 2. This property can lead to interesting applications in polarimetry and spectro-polarimetry [17], as well as holography [18] or augmented reality applications [19]. It is also interesting to point out that the GP optical elements produce a very similar splitting effect 
(but comparatively much enhanced) that chiral interfaces that can split a beam of light into its rightand left-circular polarization components. The experiment with chiral interfaces was pioneered by Fresnel almost 200 years ago and we recently revisited it with modern equipment [20].
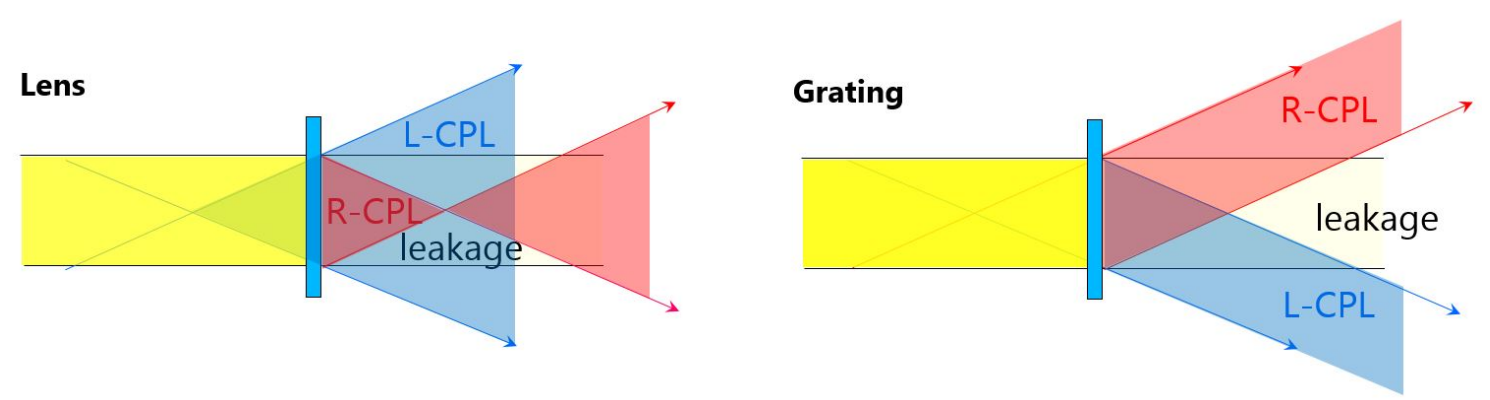

Figure 2. Schematic representation of the effect of the lens and the grating on a incoming wavefront. $\mathrm{R}-\mathrm{CPL}$ and L-CPL stand for right- and left- circularly polarizer light, respectively.

\subsection{Phase Profiles for the Grating and Lens}

The phase distribution in the plane transverse to the main propagation direction gives the wavefront structure (i.e., the phase profile) and determines the subsequent propagation behavior of the beam. Polarization gratings feature the simplest kind of inhomogeneous phase profile: a linear profile that can be expressed as

$$
\phi_{\text {grating }}(x)=\frac{\pi x}{\Lambda}
$$

where $\Lambda$ is the grating period.

Polarization lenses feature a quadratic phase function [14]:

$$
\phi_{\text {lens }}(r)=\frac{\pi}{\lambda}\left(\sqrt{f^{2}+r^{2}}-f\right),
$$

with $r$ being the radial coordinate, $f$ denoting the lens focal length, and $\lambda$ the wavelength. The GP lens has the unique property that for one circular polarization the light will focus or converge through the lens (positive focal length) and for the orthogonal circular polarization will defocus or diverge (negative focal length), as shown in Figure 2.

\section{Experiments}

The studied GP optical elements consist of a polarization lens and a polarization grating manufactured by ImagineOptix and sold by Edmund Optics. The polarization lens had a claimed focal length of $50 \mathrm{~mm}$ and dimensions of $25 \times 25 \mathrm{~mm}$ with a a thickness of $0.45 \mathrm{~mm}$. The polarization grating had the same physical dimensions as the lens and a groove density of 286 grooves $/ \mathrm{mm}(\Lambda=3.2 \mu \mathrm{m})$ that corresponds to a diffraction angle of approximately $10^{\circ}$ for $550 \mathrm{~nm}$ light.

These GP optical elements are fabricated using liquid crystal polymers that, in combination with a photo-alignment layer and a variety of chiral dopants, are engineered into three-dimensional patterns that can be captured in a thin film. The fabrication process, which is quite sophisticated, is beyond the scope of this work and it has been discussed in detail several publications $[16,18,21]$. However, as a general ideal, it is interesting to point out that these liquid-crystal based PB optical elements are basically composed of multiple twisted birefringent layers and, as we will see later, this has effects in the measured optical activity. Many other fabrication methods for GP optical elements are currently being studied, for example a new method based on ultrafast laser writing in silica glass has been recently reported [22]. 
Mueller matrix polarimetry was the optical technique used in this paper. This technique studies how the polarization properties of a material medium can transform an input Stokes vector, $\mathbf{S}_{i n}$, into an output Stokes vector $\mathbf{S}_{\text {out }}$. In most cases, this is a linear transformation that can be represented as

$$
\mathbf{S}_{\text {out }}=\mathbf{M S}_{\text {in }}
$$

where $\mathbf{M}$ is the so-called $4 \times 4$ Mueller matrix that is made of 16 real elements $m_{i j}$. Often Mueller matrices are presented normalized to the $m_{00}$ element, so that the Mueller matrix elements can only take values between -1 and 1 . The Stokes-Mueller formalism is suitable for analyzing light-medium interactions, including the case that the degree of polarization of the output beam is depolarized. However, in absence of depolarization, there is a one-to-one correspondence between Jones matrices and Mueller matrices, which means that the parameters that are contained in the Jones matrices shown in Section 2.2 can be directly extracted from the Mueller matrix [12]. In fact, several different analysis methods or decompositions have been described to study experimental Mueller matrices [23], so that it is relatively easy to obtain physical properties of a samples, such as birefringence, from a measured Mueller matrix. Generally, Mueller matrix polarimetry can be done by adding a polarization states generator (PSG) and analyzer (PSA) to the optical paths of existing non-polarization techniques, such as microscopes, thus providing complete structural information of the samples.

Polarimetric measurements that were shown in this work were made with two different home-made Mueller matrix polarimeters. The first one is a Mueller matrix microscope that uses to different rotating compensators in combination with a camera to do Mueller matrix imaging with micrometric resolution [24,25]. This instrument can work in two different modes: in the standard (or orthoscopic mode) the front focal plane of the objective is focused onto the camera sensor and different points on the camera sensor correspond to different points on the sample. In the diffractometer mode, the back focal plane of the objective is focused onto the camera sensor and so that different points on the camera sensor correspond to different directions of light diffraction [26]. The second polarimeter is a spectroscopic one and it measures the complete Mueller matrix without moving parts, because it is based on four different photoelastic modulators [27].

\section{Results}

\subsection{Phase Profiles}

The geometrical phase profiles are best studied examining the optical components with the Mueller matrix microscope as only with a polarimeter with very high spatial resolution it is possible to clearly see how the orientation of the retardation changes from point to point. Figure 3 shows the normalized Mueller matrix images measured at the central point of the lens and in the polarization grating. The lens was examined with a $10 \times$ microscope objective and the grating with a $50 \times$ objective and, in both cases, green light $(540 \mathrm{~nm})$ was used for the measurement.

From the measured Mueller matrices it is possible to determine at each pixel the orientation of the linear retardance $(\phi)$ using the analytic inversion method [12]. The in-plane orientation angle $\phi$ is given by

$$
\phi=\frac{1}{2} \operatorname{atan} 2\left(L B^{\prime}, L B\right)
$$

where $L B^{\prime}$ and $L B$ are parameters given by the analytic inversion and atan2 is the 2-argument arctangent. The results of this calculation are presented in Figure 4. As expected, the $\phi$ phase distribution forms rings of equal phase in the case of the lens and lines in the case of the grating. The phases takes values between $-90^{\circ}$ and $90^{\circ}$ and as values of $\pm 90^{\circ}$ indicate exactly the same physical orientation angle, the phase profiles of Figure 4 show discontinuities between these two values. If desired these "wrapped" profiles can be unwrapped by allowing the orientation angles to grow beyond $90^{\circ}$ as it is also shown in Figure 4. This figure also shows how the (unwrapped) experimentally measured phases compare with the simulated phases, given by Equations (12) and (11) for the lens and the grating, respectively. 
In general, there is a good agreement between them, and the small mismatches can be caused by fabrication deviations of the GP optical components from their nominal values or to the distortion introduced by the objective of our Mueller matrix microscope. When compared to other methods of studying of the geometry phase, such as polarization microscopy [28] or optical interferometry [16], Mueller matrix microscopy has the advantage that keeps the simplicity of polarization microscopy but offers fully quantitative results.
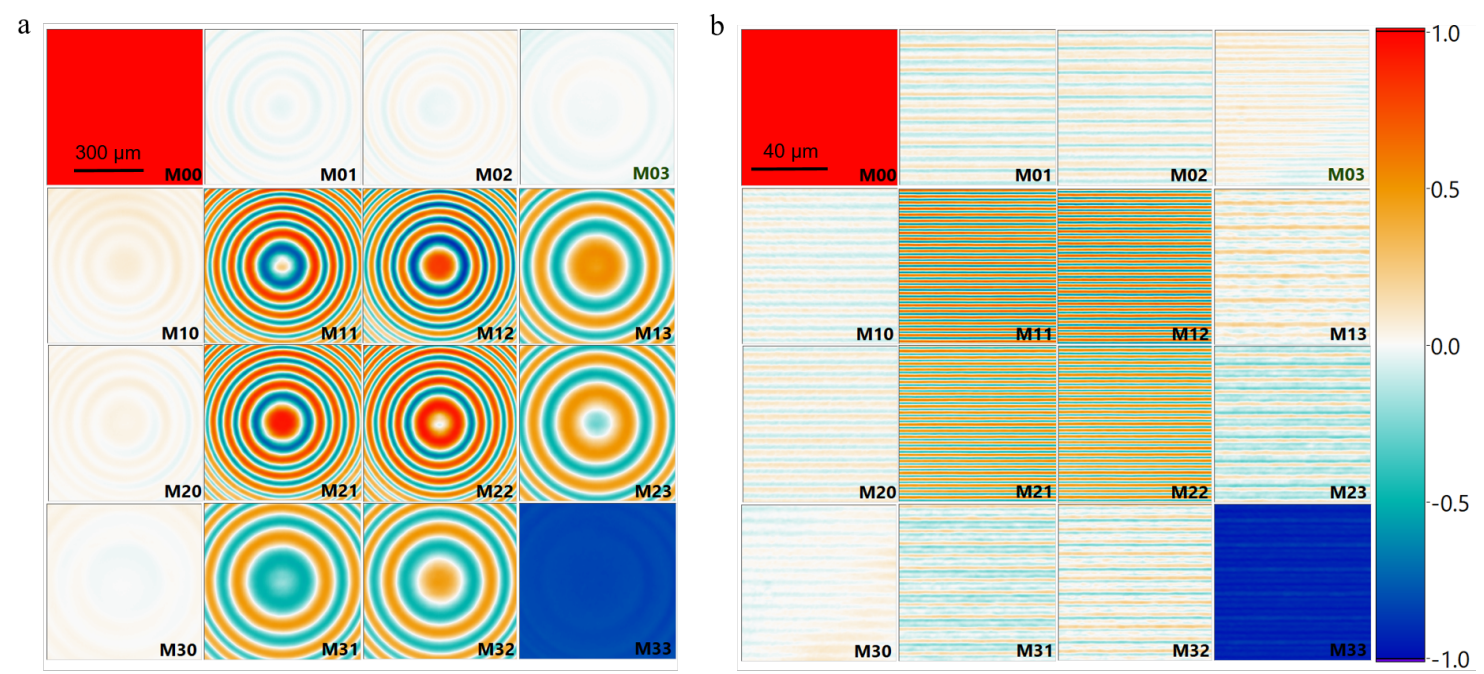

Figure 3. Normalized Mueller matrix images for the lens (a) and the grating (b). The scale bar is indicated in the $M_{00}$ element.
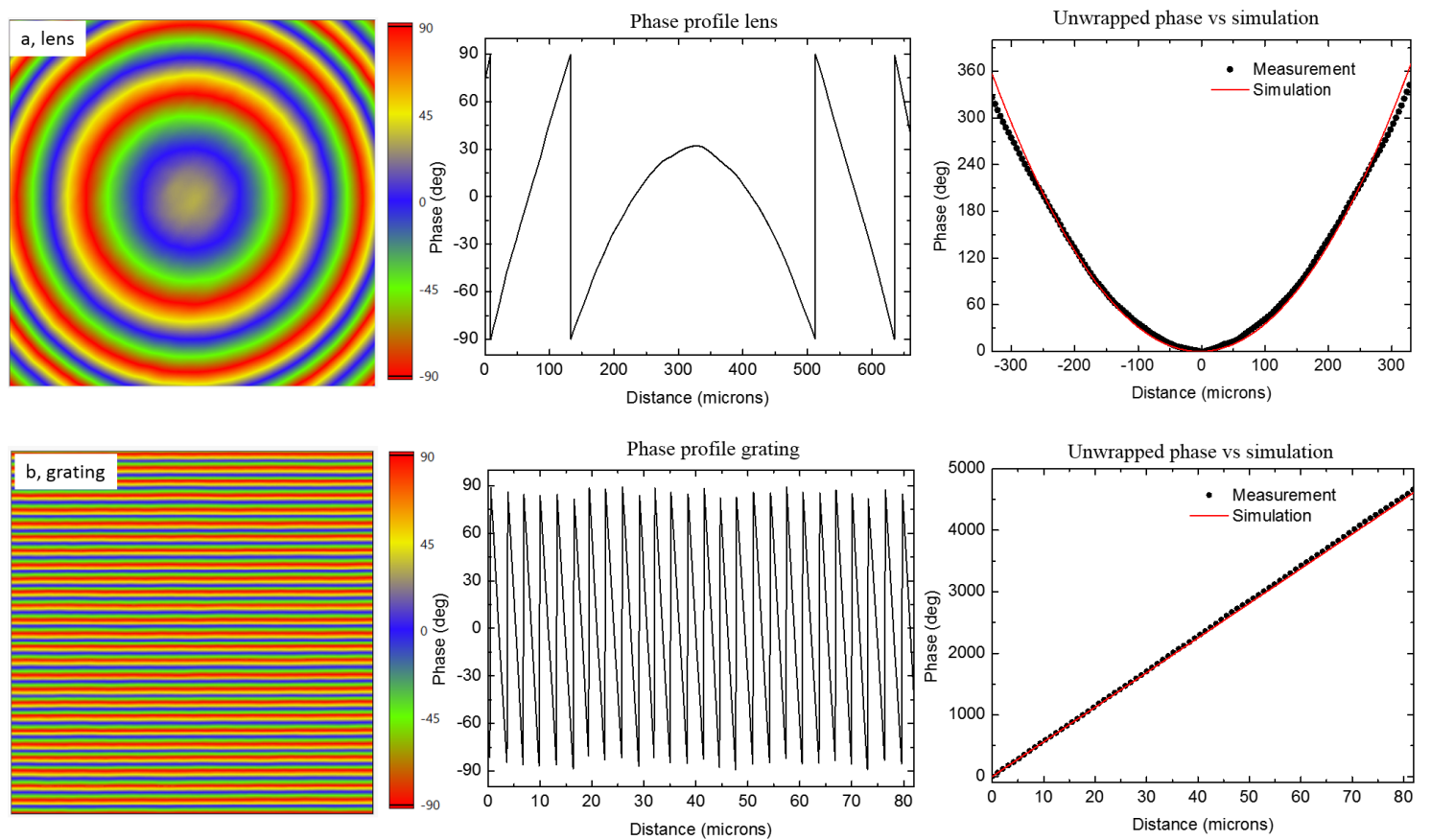

Figure 4. Phase profiles, $\phi$, for the lens (a) and the grating (b). The left pictures are the space resolved phase images, the central graphs are vertical sections of the phase profiles and the graphs on the right side show the comparison between the unwrapped phases with the simulated ones. 


\subsection{Linear and Circular Retardances}

The Mueller matrix microscopy measurements that are presented in the previous subsection, besides allowing for the calculation of the phase profiles, also provide information of the linear and circular retardances of the films. Figure 5 shows these results for the GP lens. The linear retardance has a rather homogeneous value of around $2.85 \mathrm{rad}$, slightly lower than the "ideal" value of $\pi$ that would correspond to a perfect half wave retardation. The circular retardance had, at this wavelength, a value of around $-0.3 \mathrm{rad}$ and it appeared to be slightly less homogeneous in space than the linear retardance. We also found that, while the linear retardance values remain rather constant over the whole visible range, the circular retardance values are much more wavelength, depending and they even switch signs if one compares blue and red wavelengths. This property, most likely related to the twisted nature of the liquid crystal layers, will be later discussed in more detail.

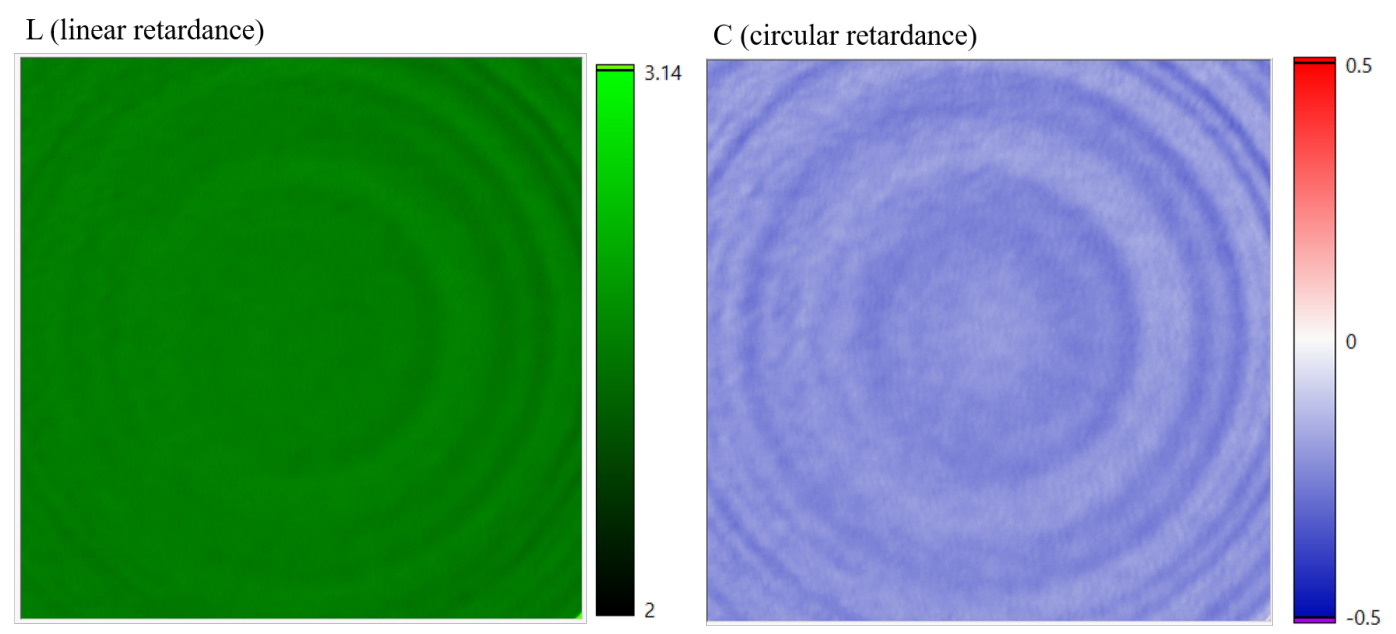

Figure 5. Magnitude values of linear and circular retardance in the central part of the lens (imaged area of $620 \mu \mathrm{m}$ by $620 \mu \mathrm{m})$. The scales are given in radians.

\subsection{Polarization Patterns of the Primary and Conjugate Waves}

A direct way to check the polarization pattern of the three waves in which the light incident on GP optical element is redistributed is using the Mueller matrix microscope in the diffractometer mode. Figure 6 shows the (unnormalized) Mueller matrix measurement made for the lens and the grating in this mode. For the measurement of the lens, the light was not incident through its center, but through a side spot. This is because a centered beam is focused/defocused by the lens, without reaching a clear separation of the waves in the space, but for side beam the separation effect is more similar to a grating.

The primary and conjugate waves are clearly distinguished in Figure 6, as they appear separated spots in the image. The leakage wave (falling in between the other two) is barely visible because it is much weaker than the other two. From the obtained images, the Mueller matrices that are associated to the primary and conjugate waves are, in good approximation:

$$
\begin{aligned}
& \left(\begin{array}{cccc}
1 & 0 & 0 & -1 \\
0 & 0 & 0 & 0 \\
0 & 0 & 0 & 0 \\
1 & 0 & 0 & -1
\end{array}\right)=\left(\begin{array}{cccc}
1 & 0 & 0 & 1 \\
0 & 0 & 0 & 0 \\
0 & 0 & 0 & 0 \\
1 & 0 & 0 & 1
\end{array}\right)\left(\begin{array}{cccc}
1 & 0 & 0 & 0 \\
0 & 1 & 0 & 0 \\
0 & 0 & -1 & 0 \\
0 & 0 & 0 & -1
\end{array}\right) \\
& \left(\begin{array}{cccc}
1 & 0 & 0 & 1 \\
0 & 0 & 0 & 0 \\
0 & 0 & 0 & 0 \\
-1 & 0 & 0 & 1
\end{array}\right)=\left(\begin{array}{cccc}
1 & 0 & 0 & -1 \\
0 & 0 & 0 & 0 \\
0 & 0 & 0 & 0 \\
-1 & 0 & 0 & 1
\end{array}\right)\left(\begin{array}{cccc}
1 & 0 & 0 & 0 \\
0 & 1 & 0 & 0 \\
0 & 0 & -1 & 0 \\
0 & 0 & 0 & -1
\end{array}\right) .
\end{aligned}
$$


In the equations above, we have shown how the measured Mueller matrix on the left can be written as the matrix product of a right- or left-circular polarizer by a half wave retarder. This is the same result that we anticipated in Equation (9), but now expressed in terms of Mueller matrices instead of Jones matrices.

Furthermore, we also verified with the spectroscopic polarimeter that these polarization properties of the primary and conjugate waves were maintained over a very wide spectral range, beyond the marketed range of $450-650 \mathrm{~nm}$.

a

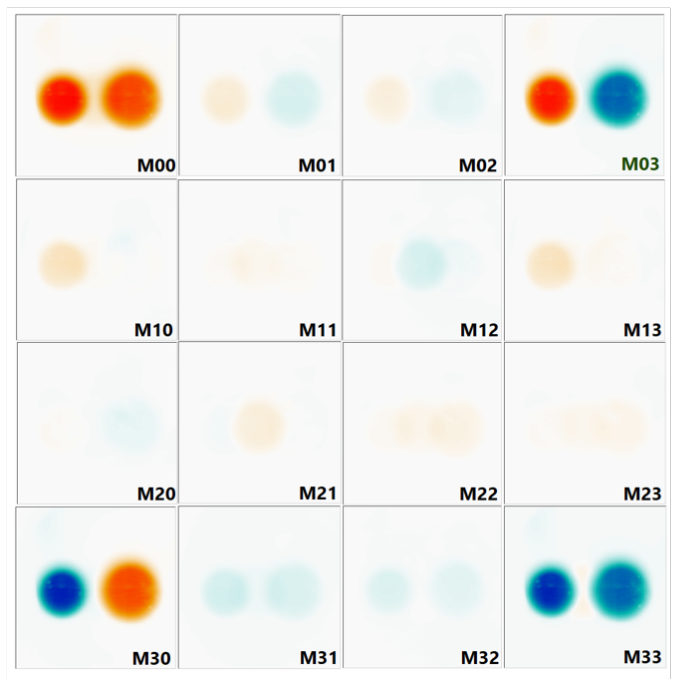

b

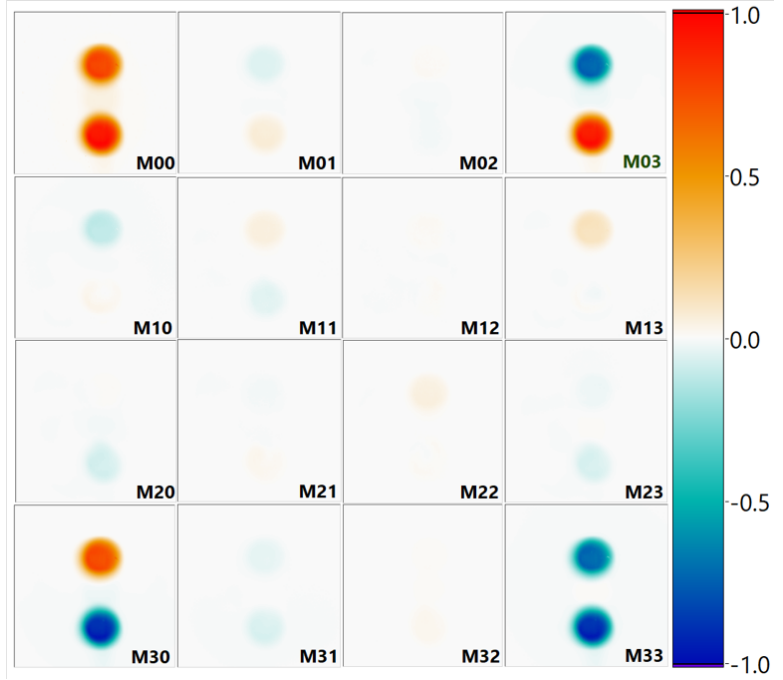

Figure 6. Unnormalized Mueller matrix images (diffractometer mode) for the lens (a) and the grating (b).

\subsection{Leakage Wave}

Both the lens and the grating gave a weak but measurable leakage wave over the whole spectral range that we were able to analyze with our polarimeters. This is unsurprising since, as it was shown in Equation (7), whenever $L \neq \pi$ and $C \neq 0$ (as it is happening in our samples, as verified in Figure 5) a leakage wave would appear. We measured the leakage wave of the grating and the lens in the interval range $400 \mathrm{~nm}$ to $800 \mathrm{~nm}$ with the spectroscopic Mueller matrix polarimeter obtaining the result that is shown in Figure 7. (Note that, in the case of the lens, the leakage wave had to be measured shining light through an edge of the lens, as if the incoming beam went through the center all three waves would remain at least partially superposed in space). As it is evident from this figure, the measured Mueller matrix is not a diagonal matrix, which would indicate that the leakage wave would preserve the polarization of the incoming wave.

The rich optical response that is shown in Figure 7 is due to the presence of circular and linear anisotropies in the measurement. In this case, as expected from the twisted nature of the liquid crystals, circular anisotropies are the largest ones, manifested not only as circular retardance, but also some circular diattenuation (that is evident here by the equal and non-vanishing values of the Mueller matrix elements $m_{03}$ and $m_{30}$ ). We believe this is most likely caused by the unequal efficiency of the primary and conjugate modes (this implies $A_{+}$being slightly different from $A_{-}$in Equation (10)), but this is something we plan to study in more detail in a future work, as here we are not measuring the efficiencies of the emerging waves. We note that the relatively small circular retardances in the optical element that are observed at a microscopic level (see Figure 6) become enhanced by several factors when only the leakage wave is examined, as shown in Figure 8. This figure displays the circular retardances determined from the analytic inversion of the Mueller matrices in Figure 7. The large 
circular retardance that is shown in Figure 8 can be more easily understood if the first Jones matrix of Equation (7), which is associated to the leakage wave, is further decomposed into two terms:

$$
\left(\begin{array}{cc}
\cos \frac{T}{2} & \frac{C}{T} \sin \frac{T}{2} \\
-\frac{C}{T} \sin \frac{T}{2} & \cos \frac{T}{2}
\end{array}\right)=\frac{C}{T}\left(\begin{array}{cc}
\cos \frac{T}{2} & \sin \frac{T}{2} \\
-\sin \frac{T}{2} & \cos \frac{T}{2}
\end{array}\right)+\frac{T-C}{T} \cos \frac{T}{2} \mathbf{I}
$$

where $\mathbf{I}$ is the $2 \times 2$ identity matrix. The first term of this decomposition is a pure rotator Jones matrix, while the second one corresponds to an identity matrix. Thus, in the presence of circular retardance $(C \neq 0)$, the leakage wave will be formed by the superposition of two waves, one with the polarization of the input but rotated by $\frac{T}{2}$ and another one keeping the input polarization. As, in a GP optical component, $T$ is generally quite close to $\pi$ (because $L \simeq \pi$ and $C$ is small), the second term of Equation (17) may become vanishing small, so that the first rotator term is still relevant in the leakage wave, even for relatively small values of $C$.

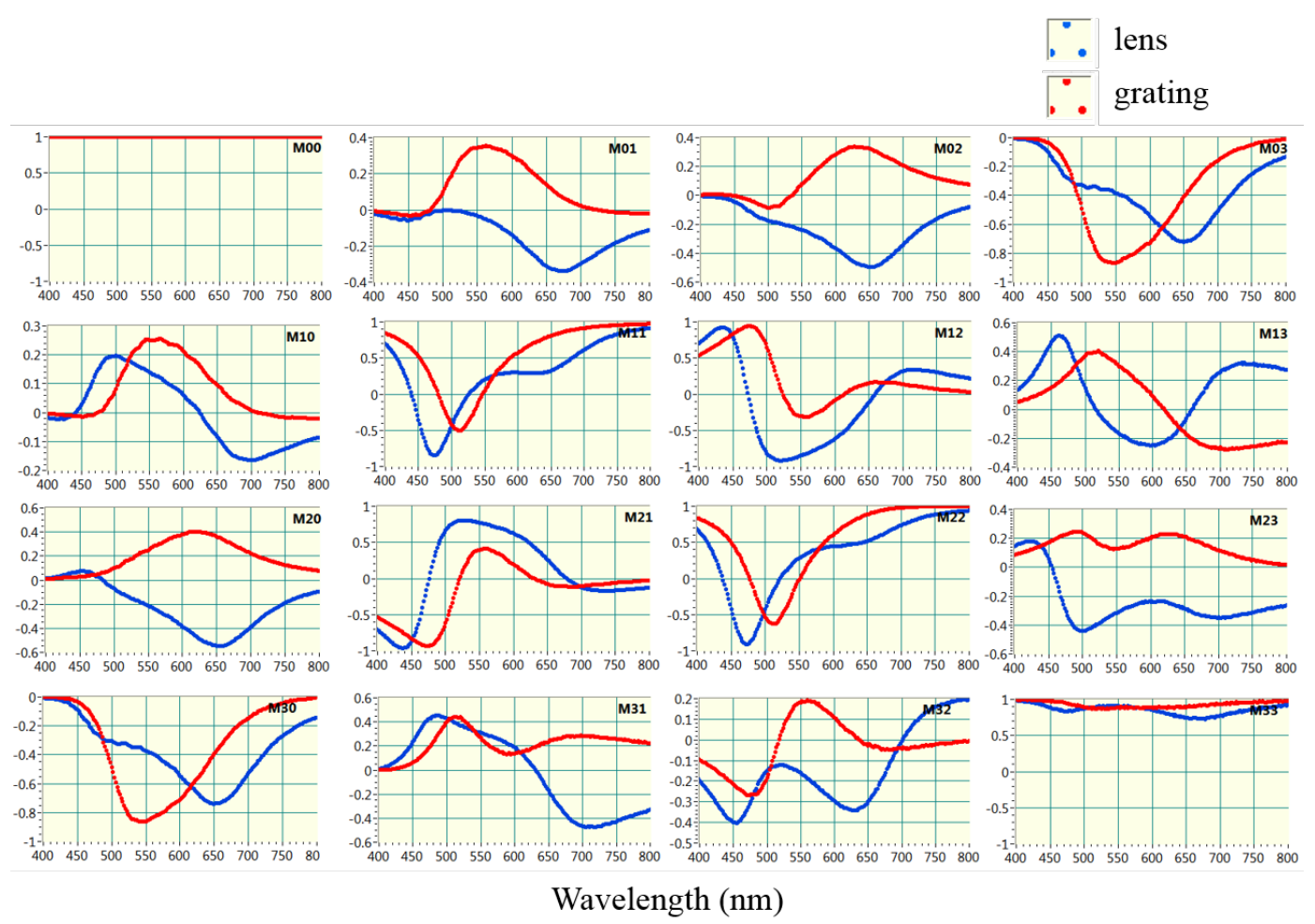

Figure 7. Spectroscopic Mueller matrices measured for the leakage wave of the lens and the grating. All of the Mueller matrix elements have been normalized to the element $m_{00}$.

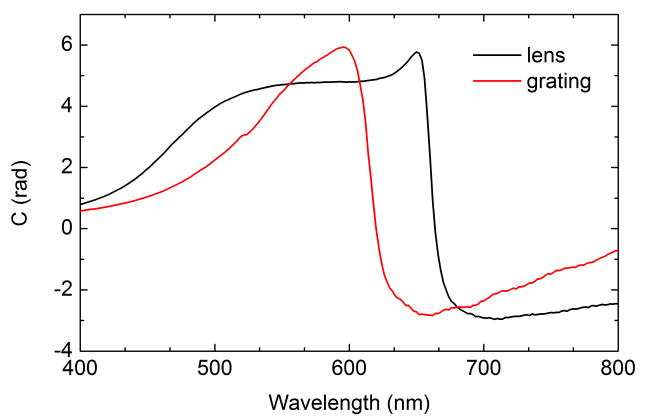

Figure 8. Circular retardance measured from the leakage wave of the lens and the grating. 
The existence of linear anisotropies in the leakage wave (i.e. the fact that the polarization of the leakage wave changes if the GP optical element is azimuthally rotated) was not anticipated in Equation (17), because, as shown there, the Jones matrix associated to the leakage wave includes only circular anisotropies. The reasons for this response are not yet fully clear, but we think they are most likely caused by the non-perfect spatial homogeneity of the magnitude of $L$ over the illumination spot, as was already observed in Figure 6.

From the discussion above, it is quite clear that the polarimetric measurement of the leakage wave is a very sensitive and straightforward method to study how "ideal" the GP optical element is. We suggest that it is should be taken into consideration in the future evaluation of GP optical elements, as it only implies a rather simple and non-destructive measurement.

\section{Conclusions}

We have demonstrated that the properties GP optical elements can be well studied using a polarimetric technique without need to resort to the interferometric methods that are typically needed to quantify dynamical phases. The phase profiles can be directly obtained from Mueller matrix measurements with micrometric spatial resolution. We have also shown, both theoretically and experimentally, that the deviation of linear retardance from the half-wave condition and the presence of circular retardance contributes to the existence of a non-negligible leakage wave that affects the overall efficiency of the optical element. However, even in these circumstances, the polarization of the primary and conjugate waves is fully circularly polarized regardless of the polarization input beam, as the space variant linear retardation is essentially acting as a polarization beam-splitter. Our results also indicate that the measurement of Mueller matrix associated to the leakage wave (technically, it is the simplest measurement to do because there is no redirection of the wavefront) is particularly informative about various aspects of the optical element such as the presence of circular retardance, the presence of linear and circular diattenuating losses or the existence of an unequal efficiency for the primary and conjugate waves.

The technological progress in the fabrication of GP optical components is making possible a new generation of gratings, lenses, and other elements that are physically thin and have very small losses. We think that polarimetry is the ideal technique to study and benchmark these new components.

Author Contributions: O.A. designed the research and the methodology used, did part of the experimental measurements and participated in their analysis and interpretation and wrote the manuscript. H.B. did part of the experimental measurements and participated in their analysis and interpretation. Both authors have revised the manuscript. All authors have read and agreed to the published version of the manuscript.

Funding: This work was funded by Spanish Ministerio de Ciencia, Innovación y Universidades through projects with grant numbers RTI2018-098410-J-I00, CTQ2017-87864-C2-1-P and RYC2018-024997-I.

Conflicts of Interest: The authors declare no conflict of interest.

\section{References}

1. Forbes, A. Structured Light: Tailored for Purpose. Opt. Photonics News 2020, 31, 24-31. [CrossRef]

2. Lee, G.Y.; Sung, J.; Lee, B. Recent advances in metasurface hologram technologies. ETRI J. 2019, 41, 10-22. [CrossRef]

3. Gerislioglu, B.; Dong, L.; Ahmadivand, A.; Hu, H.; Nordlander, P.; Halas, N.J. Monolithic metal dimer-on-film structure: New plasmonic properties introduced by the underlying metal. Nano Lett. 2020, 20, 2087-2093. [CrossRef]

4. Gerislioglu, B.; Bakan, G.; Ahuja, R.; Adam, J.; Mishra, Y.K.; Ahmadivand, A. The role of Ge2Sb2Te5 in enhancing the performance of functional plasmonic devices. Mater. Today Phys. 2020, 12, 100178. [CrossRef]

5. Pancharatnam, S. Generalized theory of interference and its applications. In Proceedings of the Indian Academy of Sciences-Section A; Springer: Berlin/Heidelberg, Germany, 1956; Volume 44, pp. 398-417.

6. Berry, M. Pancharatnam, virtuoso of the Poincaré sphere: An appreciation. Curr. Sci. 1994, 67, $220-223$. 
7. Berry, M.V. The adiabatic phase and Pancharatnam's phase for polarized light. J. Mod. Opt. 1987, 34, 1401-1407. [CrossRef]

8. Bhandari, R. Polarization of light and topological phases. Phys. Rep. 1997, 281, 1-64. [CrossRef]

9. Bomzon, Z.; Biener, G.; Kleiner, V.; Hasman, E. Space-variant Pancharatnam-Berry phase optical elements with computer-generated subwavelength gratings. Opt. Lett. 2002, 27, 1141-1143. [CrossRef]

10. Roux, F.S. Geometric phase lens. JOSA A 2006, 23, 476-482. [CrossRef]

11. Marrucci, L.; Manzo, C.; Paparo, D. Pancharatnam-Berry phase optical elements for wave front shaping in the visible domain: Switchable helical mode generation. Appl. Phys. Lett. 2006, 88, 221102. [CrossRef]

12. Arteaga, O.; Canillas, A. Analytic inversion of the Mueller-Jones polarization matrices for homogeneous media. Opt. Lett. 2009, 35, 559-561. [CrossRef] [PubMed]

13. Arteaga, O. Mueller Matrix Polarimetry of Anisotropic Chiral Media. Ph.D. Thesis, Universitat de Barcelona, Barcelona, Spain, 2010.

14. Yu, H.; Zhou, Z.; Qi, Y.; Zhang, X.; Wei, Q.H. Pancharatnam-Berry optical lenses. J. Opt. Soc. Am. B 2019, 36, D107-D111. [CrossRef]

15. Hasman, E.; Bomzon, Z.; Niv, A.; Biener, G.; Kleiner, V. Polarization beam-splitters and optical switches based on space-variant computer-generated subwavelength quasi-periodic structures. Opt. Commun. 2002, 209, 45-54. [CrossRef]

16. Kim, J.; Li, Y.; Miskiewicz, M.N.; Oh, C.; Kudenov, M.W.; Escuti, M.J. Fabrication of ideal geometric-phase holograms with arbitrary wavefronts. Optica 2015, 2, 958-964. [CrossRef]

17. Acher, O.; Richard, S. Easy-to-build Wollaston-like polarization splitter with adjustable beam deviation and tunable chromatic dispersion. Opt. Express 2019, 27, 29232-29240. [CrossRef]

18. Xiang, X.; Kim, J.; Escuti, M.J. Far-field and fresnel liquid crystal geometric phase holograms via direct-write photo-alignment. Crystals 2017, 7, 383. [CrossRef]

19. Cui, W.; Chang, C.; Gao, L. Development of an ultra-compact optical combiner for augmented reality using geometric phase lenses. Opt. Lett. 2020, 45, 2808-2811. [CrossRef]

20. Arteaga, O.; Garcia-Caurel, E.; Ossikovski, R. Stern-Gerlach experiment with light: Separating photons by spin with the method of A. Fresnel. Opt. Express 2019, 27, 4758-4768. [CrossRef]

21. Komanduri, R.K.; Lawler, K.F.; Escuti, M.J. Multi-twist retarders: Broadband retardation control using self-aligning reactive liquid crystal layers. Opt. Express 2013, 21, 404-420. [CrossRef]

22. Sakakura, M.; Lei, Y.; Wang, L.; Yu, Y.H.; Kazansky, P.G. Ultralow-loss geometric phase and polarization shaping by ultrafast laser writing in silica glass. Light. Sci. Appl. 2020, 9, 1-10. [CrossRef]

23. Perez, J.J.G.; Ossikovski, R. Polarized Light and the Mueller Matrix Approach; CRC Press: Boca Raton, FL, USA, 2016.

24. Arteaga, O.; Baldrís, M.; Antó, J.; Canillas, A.; Pascual, E.; Bertran, E. Mueller matrix microscope with a dual continuous rotating compensator setup and digital demodulation. Appl. Opt. 2014, 53, 2236-2245. [CrossRef] [PubMed]

25. Kuntman, E.; Arteaga, O.; Antó, J.; Cayuela, D.; Bertran, E. Conversion of a polarization microscope into a Mueller matrix microscope. Application to the measurement of textile fibers. Opt. Pura Apl. 2015, 48, 309-316. [CrossRef]

26. Arteaga, O.; Nichols, S.M.; Antó, J. Back-focal plane Mueller matrix microscopy: Mueller conoscopy and Mueller diffractrometry. Appl. Surf. Sci. 2017, 421, 702-706. [CrossRef]

27. Arteaga, O.; Freudenthal, J.; Wang, B.; Kahr, B. Mueller matrix polarimetry with four photoelastic modulators: theory and calibration. Appl. Opt. 2012, 51, 6805-6817. [CrossRef] [PubMed]

28. Piccirillo, B.; Picardi, M.F.; Marrucci, L.; Santamato, E. Flat polarization-controlled cylindrical lens based on the Pancharatnam-Berry geometric phase. Eur. J. Phys. 2017, 38, 034007. [CrossRef]

(C) 2020 by the authors. Licensee MDPI, Basel, Switzerland. This article is an open access article distributed under the terms and conditions of the Creative Commons Attribution (CC BY) license (http:/ / creativecommons.org/licenses/by/4.0/). 